\title{
La planeación didáctica como factor determinante en la autoeficacia del maestro universitario
}

\author{
Patricia Islas Salinas \\ María Olivia Trevizo Nevárez \\ Alberto Heiras Torres
}

Profesores investigadores Universidad Autónoma de Ciudad Juárez, División Cuauhtémoc Instituto Tecnológico de Ciudad Cuauhtémoc

\section{Resumen}

n los últimos años el papel del pro$\checkmark$ fesor universitario se ha transfor$\checkmark$ mado, tanto en las características formales de su educación como en las funciones que realiza; la mayoría de los catedráticos universitarios no poseen una formación docente, son profesionistas que en un momento de su vida laboral asumen el rol de catedráticos, esto implica diferentes percepciones de autoeficacia en su práctica docente. Se observa en los resultados de esta investigación que aunque las bases docentes se le proporcionan en la capacitación institucional, no fortalecen del todo su autoeficacia, que aunque en este caso se considera aceptable, una de las dimensiones en las que más falla es en el aspecto de la evaluación. En lo referente a las dimen- siones del diseño de objetivos, autorregulación y diseño de la planeación, coinciden en que se tiene la capacidad e intención pero en el momento de la ejecución tienen fallas mínimas.

Palabras clave: percepciones, docentes, autoconcepto, planificación.

\section{Introducción}

El propósito de la presente investigación es analizar e interpretar las percepciones de los docentes universitarios sobre la autoeficacia y su relación con factores que inciden en la práctica pedagógica en particular la planeación didáctica.

En este estudio se observa como el docente universitario planea sus actividades diarias, así como la ejecución y evaluación 
de las mismas, evidenciando un perfil similar al de los profesores de todas las universidades del país.

Las creencias de autoeficacia son concepciones propias del docente que lo llevan a actuar de una manera determinada en la búsqueda del logro de las metas impuestas por el mismo y por la institución a la que pertenece en pro del aprendizaje de los estudiantes. Chacón (2006) plantea que la autoeficacia percibida por el docente afecta de manera positiva o negativa el ambiente de aprendizaje así como también la eficiencia y el auto concepto docente.

El profesor universitario debe reflexionar sobre su autoeficacia para asumir nuevos roles en la implementación de estrategias, habilidades y utilización de recursos. Lo anterior nos lleva a implicar algunos factores que pueden incidir en la percepción de autoeficacia en los docentes, uno de estos factores es la planeación didáctica ya que esta apoya lo que se quiere llevar a la práctica en el aula aun cuando no siempre se lleve a cabo fielmente, pero el hecho de elaborar esta planeación antes del inicio de un curso proporciona al docente universitario seguridad y claridad en sus acciones durante el transcurso del periodo de clases.

Con esta investigación se pretende demostrar la importancia de la planificación didáctica en el sentimiento de autoeficacia de los docentes universitarios, debido a que influye significativamente en la práctica docente, Prieto (2005) demostró que los profesores con un elevado sentimiento de autoeficacia están más dispuestos a innovar, a correr riesgos probando estrategias nuevas, manifiestan más compromiso y entusiasmo por ser docentes.

La autoeficacia tiene que ver con la percepción que una persona tiene de sí misma y sobre la capacidad para realizar sus funciones de manera que esto le proporcione un sentimiento de seguridad; Bandura (1997) plantea que los individuos poseen un sistema interno propio que les capacita para ejercer control sobre sus pensamientos, sentimientos, motivaciones y conductas. Este sistema interno, proporciona a la persona un mecanismo de referencia que es la base sobre la cual percibimos, regulamos y evaluamos nuestra conducta (Gonzalo y León, 1999).

La reflexión sobre la práctica docente se determina en las acciones educadoras que se dan en los ambientes de aprendizaje, la planeación didáctica es una herramienta que el docente puede utilizar para que estas acciones sean efectivas, ya que al planear las estrategias, técnicas y recursos que van a apoyarlo en el aula para lograr los objetivos, habilidades y competencias que se pretende desarrollar en los estudiantes, el docente universitario conseguirá un alto sentimiento de autoeficacia.

Lule (2003) señala que la práctica docente se compone de actividades de enseñanza y aprendizaje que se desarrollan en el aula, de acuerdo con una planificación e incluyen el apoyo a los alumnos en torno a un contenido programático.

La planeación didáctica puede ser flexible, el docente con esta herramienta puede abrir su mentalidad a la posibilidad de edificar sobre lo planeado, es decir, tiene la ca- 
pacidad de reflexionar antes del momento de enfrentarse a los estudiantes en el aula y "moldear" su planeación de acuerdo al contexto y ambiente que ocurre en la misma, pues como lo saben quienes se dedican a la docencia, el sistema áulico, es un sistema vivo, con características propias y diferente a los demás, porque este ambiente es edificado por las personas que forman un grupo, dándole una personalidad y vida disímil, aun cuando se trate de grupos de estudiantes diferentes con la misma asignatura y en una misma universidad.

\section{Estrategia metodológica}

Para la adquisición de datos en sujetos donde la percepción, opiniones y sentimientos tienen mucha importancia, se diseñó un procedimiento donde los datos tanto cuantitativos como cualitativos se recaban en un mismo instrumento, ésta concepción pragmática del conocimiento, permite al investigador "abrirse a métodos múltiples, diferentes puntos de vista del mundo y diferentes supuestos, así como diferentes formas de obtener y analizar datos" (Guzmán y Alvarado, 2009).

\section{Método de investigación mixto}

Los métodos mixtos permiten mayor flexibilidad porque se adaptan a las necesidades del investigador y se obtienen tanto resultados cuantitativos como cualitativos por lo que lo más viable es aplicar instrumentos que evalúen las diferentes aspectos mencionados anteriormente, de los cuales algunos no son medibles y se requieren datos sobre opiniones, sentimientos y formas de pensar.

\section{Instrumentos y técnicas para recabar datos}

Se utilizó una adaptación de la escala que Leonor Prieto (2007) aplicó para medir la autoeficacia del profesor Universitario en un estudio realizado en Narcea, España.

La adaptación consiste en cinco preguntas abiertas relacionando la autoeficacia del docente con la planeación didáctica, en la segunda parte del instrumento el docente contesta 15 preguntas con una escala del 1 al 5, donde responde sobre la capacidad para realizar cada tarea relacionada con su planeación y las mismas preguntas acerca de la regularidad con que realiza dichas actividades, con una escala del 1 al 5.

En la tercera parte el docente se autoevalúa haciendo una sumatoria de su puntuación.

\section{Sujetos investigados}

La investigación se llevó a cabo con docentes universitarios pertenecientes a dos de las instituciones más importantes en la región de Cuauhtémoc, Chih., México: la Universidad Autónoma de Ciudad Juárez, división Cuauhtémoc; y el Instituto Tecnológico de Ciudad Cuauhtémoc.

\section{Análisis y Resultados}

Percepciones de docentes universitarios sobre la planeación didáctica como factor en la autoeficacia

La autoeficacia es la creencia de que uno tiene las habilidades para ejecutar los cursos de acción requeridos, corrigiendo el camino para lograr los objetivos planea- 
dos, siendo eficientes mediante motivación propia, confiando en las propias potencialidades y capacidades, aprendiendo a manejar el tiempo y el trabajo en función de la visión, misión y las metas propias, a través de la disciplina adquiriendo herramientas y métodos para el trabajo, realizando las tareas de manera efectiva para ser capaz de llegar al éxito.

Esta concepción rescatada de los encuestados indica que la autoeficacia es un juicio de sí mismo sobre sus habilidades y capacidades para realizar una tarea que los lleve al buen ejercicio docente en el aula.

Considerando que la autoeficacia es innata en la medida que se tiene la predisposición natural a enfrentar retos y alcanzarlos, sin embargo, también puede aprenderse a pesar de las creencias acerca de las capacidades y habilidades personales, las cuales se construyen durante el crecimiento mediante el contacto social iniciando en el contexto familiar.

La mayoría de los conceptos que llevan el prefijo auto proviene del griego "autos" que significa por o en sí mismo, por ejemplo: autoestima, autoimagen etc., queriendo hacer referencia a la psicología del sí mismo, Roger (citado por Aquilino, 2003) lo refiere como el conjunto integrado de elementos que el individuo construye acerca de él mismo ya que cada individuo experimenta la realidad de un modo único y singular, afectando esto, la percepción de autoeficacia. El término personalidad se refiere al "desempeño de la persona", así habrá personas que tienen una personalidad melancólica, entusiasta, etc., estos rasgos de personalidad son innatos, se acentúan con el correr de los años y por la influencia del medio ambiente. Con lo dicho anteriormente podemos decir que la autoeficacia está relacionada en cierta manera con la personalidad individual ya que ésta, al creer que se cuenta con las habilidades necesarias para proponer y ejecutar cursos de acción se define según los rasgos de personalidad individuales. En otras palabras, la autoeficacia se puede aprender y adquirirá "un sabor distintivo" según el tipo de personalidad individual.

\section{Planeación didáctica como elemento para reforzar la autoeficacia}

La planeación didáctica es la parte medular en la propuesta de enseñanza del docente, es un instrumento para organizar las actividades docentes en el aula y para cumplir los objetivos que lleven al aprendizaje.

Si la autoeficacia es la creencia de que se tienen las habilidades para ejecutar los cursos de acción requeridos en el manejo de situaciones áulicas previstas, la planeación es el mapeo del cómo se habrán de ejecutar dichos cursos de acción. La planeación es necesaria porque lleva al éxito las actividades.

Una buena planeación didáctica aumenta las posibilidades y la frecuencia de obtener un sentimiento de autoeficacia constante, por lo tanto existe una correlación directa entre planeación didáctica y autoeficacia, es decir, si la primera no se lleva a cabo, la segunda no se alcanza en su totalidad. 
La evaluación que realizan los estudiantes a los docentes es un elemento sumamente importante, pues el nivel de autoeficacia percibida afecta el esfuerzo, la dedicación, metas y compromisos de los docentes en su práctica. Los estudiantes también perciben cuando los docentes han llevado una planificación didáctica de las actividades áulicas. Según Ortega, Rosales, y Sánchez (2011) investigadores de la autoeficacia docente encuentran que el 83\% de los docentes perciben su autoeficacia en relación directa con la preparación de los recursos didácticos más adecuados para desarrollar las actividades escolares, resultados muy similares a los encontrados en esta investigación.

El programa institucional de formación docente 2012, plantea una acción formadora nombrada Programa de capacitación y desarrollo del personal académico que pretende una formación integral y la adquisición de competencias para el desempeño laboral bajo el enfoque educativo centrado en el aprendizaje.

De igual manera las instituciones que nos ocupan poseen programas institucionales de formación y capacitación continua para sus docentes de acuerdo al modelo pedagógico que utilizan.

Análisis de datos obtenidos con la escala de autoeficacia en relación a la planeación didáctica

A partir del análisis de los datos obtenidos con el instrumento, se rescataron cuatro dimensiones: objetivos, evaluación, diseño y autorregulación.

\section{Primera dimensión de análisis: Objetivos}

"Al definir los objetivos se explica el resultado que se pretende obtener como resultado de este proceso educativo, dando mayor importancia a lo que hará al educando y no lo que hará la institución" (Aguilar y Vargas, 2010).

A partir de las preguntas del instrumento utilizado, los objetivos se identifican como una dimensión de análisis. La mayoría de los sujetos especifican a sus estudiantes los objetivos que se pretende alcanzar, además identifican claramente los propósitos de la temática estudiada en cada clase. De lo anterior se deduce que los docentes universitarios identifican, especifican y plasman en la planeación didáctica los objetivos semestrales del curso.

\section{Segunda dimensión de análisis:}

\section{Diseño}

El diseño de instrumentos de evaluación acorde a los objetivos, la estructura de cada clase, la utilización de estrategias, técnicas de aprendizajes y recursos didácticos, constituye lo medular para la planeación didáctica. El docente selecciona y adapta cada uno de ellos de acuerdo a sus necesidades.

Los datos obtenidos muestran que la mayoría de los docentes universitarios se sienten capaces de diseñar los diferentes elementos que conforman una planeación, pero no lo realizan previo a la clase. Esto indica que tienen un alto sentimiento de autoeficacia en el diseño y menor medida en la ejecución. 
REVISTA DE INVESTIGACIÓN EDUCATIVA DE LA REDIECH N. 9

Tercera dimensión de análisis: Autorregulación

En el análisis las siguientes categorías forman parte de la autorregulación del docente universitario:

- preparar material,

- adaptarse a las necesidades de los estudiantes,

- actualizar sus conocimientos para dominar el contenido,

- tener un pensamiento flexible al momento de instrumentar la planeación $\mathrm{y}$

- darse tiempo de planificar.

Los resultados nos dicen que el docente universitario se siente capaz de hacerlo pero no se da el tiempo de ejecutarlo. Están conscientes de sus capacidades por su formación profesional, pero en la medida en que no corresponde al área docente esto incide en la disposición de ejecutar las actividades que tienen que ver con algunas de las categorías arriba mencionadas, por lo que requieren de un esfuerzo extra para empatizar con los estudiantes, entender sus necesidades y ajustarlas a la planeación, esto les genera un sentimiento de autoeficacia menor.

Cuando un docente tiene la capacidad de modificar sus pensamientos y adaptar su planeación a las necesidades de la clase, de tal manera que actualiza sus planes cuando se percata de que la planeación no resultó adecuada para el objetivo deseado, es decir a partir de un pensamiento flexible practica la autorregulación. De acuerdo con Schoenfeld (citado por García-Cabrero,
Loredo y Carranza, 2008) los procesos que ocurren antes de la acción didáctica dentro del aula, como la planeación, se actualizan constantemente durante la interacción con los propios contenidos, así como con los alumnos.

\section{Cuarta dimensión de análisis: Evaluación}

La evaluación es una dimensión que abarca en el instrumento las categorías de análisis siguientes: instrumentos necesarios para evaluar el aprendizaje y los criterios que se utilizarán durante el semestre en cada módulo. Planean la evaluación y los criterios de la misma pero no la llevan a cabo del todo, se observa una discrepancia entre lo planeado y lo ejecutado.

A menudo el docente define sus criterios de evaluación sin tomar en cuenta el currículo, por lo que según Martínez (2011) es fácil comprobar cómo algún docente "inventa" estos criterios hasta el punto de desvirtuar la evaluación que propone al alumno; estas malas prácticas evaluadoras se han venido realizando durante mucho tiempo, como resultado de la falta de capacitación del docente en este rubro y al parecer es este el más complicado elemento dentro de la planeación didáctica, esto indica que es necesario ajustar la evaluación del alumnado a la normativa curricular y de la evaluación referida por las políticas educativas de cada institución.

Por otro lado, el docente tiene la decisión y el poder de determinar los criterios e instrumentos de evaluación, lo cual lo hace que asuma actitudes protagónicas y autoritarias ante el estudiante. 


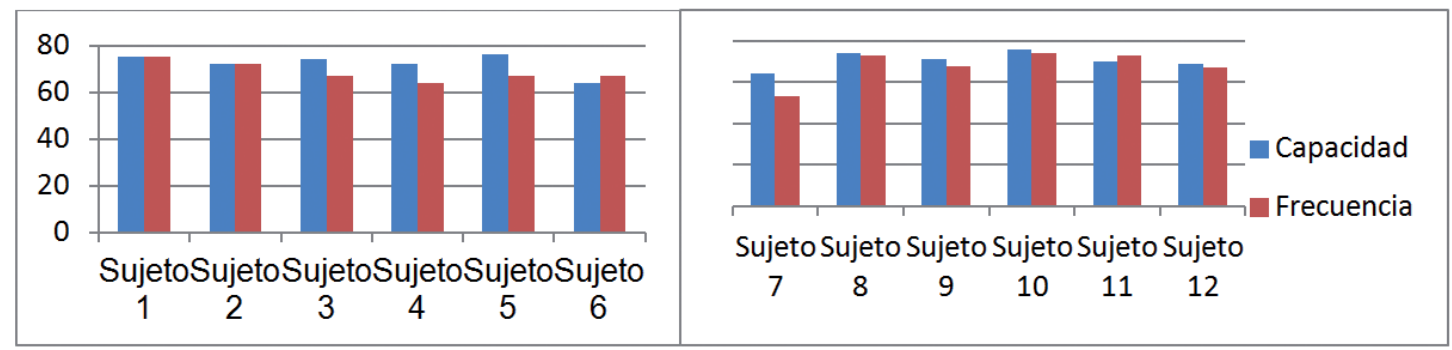

Figura 1

La evaluación es uno de los procesos finales de la planeación, el docente llega agotado a esta fase y por ende le dedica poco tiempo y atención, a diferencia de las otras dimensiones, es aquí donde otras de las dimensiones pueden ser de utilidad como la autorregulación ya que el docente en estas instituciones tiene la libertad de decidir cómo, en qué momento y con qué criterios evaluará.

\section{Resultados generales}

Clark y Jinger (citados por Monroy, 1998) señalan que la planeación didáctica implica procesos psicológicos en donde el docente manifiesta el deseo de que ocurra algo y además ayuda a que ese deseo se convierta en acción.

Existe un conflicto muy serio en los docentes entre planear o improvisar, los que planean manifiestan el deseo de ejecutar las acciones para tener los resultados esperados, pero entre el deseo y la ejecución existe un vacío.

La mayoría de los docentes encuestados, se sienten capaces de realizar una planeación didáctica y están conscientes de que esto es un factor que determina la autoeficacia, sin embargo, en el momento de la realización y ejecución de la mismas, demuestran según sus respuestas que su nivel de compromiso para hacerlo de manera frecuente es más bajo, sin embargo están por encima del mínimo y encima del promedio (fig. 1).

En los resultados de esta investigación encontramos, cómo la evaluación es una de las dimensiones donde el docente se percibe menos auto eficaz.

\section{Conclusiones}

Los docentes universitarios en las instituciones que refieren a esta investigación se caracterizan por ser un colectivo amplio, diverso y seriamente problematizado, misma situación que se enfrenta en todo el país, según Hernández y Pérez (2011) esto puede deberse a que la mayoría de los catedráticos en las universidades son profesionistas sin formación docente, que no cuentan con herramientas didácticas y pedagógicas para su labor en el aula, amén de las que la propia institución les proporciona a través de capacitaciones en el modelo pedagógico en que cada una está inserta.

Según estudios de ANuies (2000) y Gil (1994-1998) entre otras, determinan que el perfil predominante de los docentes universitarios mexicanos continúa siendo tradicionalista, el $77 \%$ de los docentes comienzan su trabajo académico sin experiencia previa y cerca de un $80 \%$ admite 
contar con una preparación didáctica deficiente; sin embargo, aquellos docentes que trabajan en estas instituciones y se consideran auto eficaces, tal vez por su actitud y compromiso ante las mismas o por su simple percepción, practican la autorregulación para adquirir las competencias docentes que necesitan para otorgar una educación de calidad a los estudiantes, cabe mencionar que la mayoría de los maestros encuestados se consideran capaces de realizar una planeación didáctica pero al momento de ejecutarla dentro del aula su sentido de autoeficacia disminuye.

Dentro de los aspectos menos favorecedores de la autoeficacia docente están todos aquellos que se refieren a los procesos de evaluación, según Díaz y Rigo (2003) este es el proceso en el cual los docentes se involucran menos.

Las dimensiones analizadas en esta investigación (objetivos, diseño, autorregulación y evaluación) como parte fundamental de la planeación didáctica indican que esta es un factor importante en la percepción de la autoeficacia.

\section{Referencias}

Aguilar, J. y Vargas, J. (2010). Planeación educativa y diseño curricular: un ejercicio de sistematización. Asociación Oaxaqueña de Psicología A.C. / Centro Regional de Investigación en Psicología. México. En: http://www.conductitlan.net/notas_boletin_investigacion/140_planeacion_educativa_curriculum.pdf.

Aquilino, P. (2003). Fundamentos de la Psicología de la Personalidad. Ed. Rialp. España.

Bandura, A. et al. (1997). Test of generality of self efficacy theory. Cognitive Therapy and research. 4, p. 39-66.

Chacón, C. (2006). Las creencias de autoeficacia: un aporte para la formación docente de inglés. Acción pedagógica. 15. 44-54. En: http://www.saber.ula.ve/ bitstreamo/123456789/17262/2/articulo5.pdf

Díaz, F. y Rigo, M. (2003). Realidades y paradigmas de la función docente: implicaciones sobre la evaluación magisterial en la educación superior. Revista de educación superior. Vol. XXXII (3). $\mathrm{N}^{\circ} 127$. México.

García-Cabrero, B., Loredo, J. y Carranza, G. (2008). Análisis de la práctica educativa de los docentes: pensamiento, interacción y reflexión. Revista Electrónica de Investigación Educativa, Especial. En: http://redie.uabc.mx/NumEsp1/contenido-garcialoredocarranza.html

Gil, A. (1994). Los rasgos de la diversidad: un estudio sobre los académicos mexicanos. UAM. México.

(1998). Origen, conformación y crisis de los informadores mexicanos: posibilidades y límites de una reforma en curso. Tres décadas de políticas del estado en educación superior. Anuies. México.

Gonzalo, M. y León, B. (1999). La Promoción de la Autoeficacia en el Docente Universitario. Revista Electrónica Interuniversitaria de Formación del Profesorado, 2 (1). Asociación Universitaria de Formación del Profesorado. En: http://www.uva.es/autofop/publica/revelfop/99-v2n1.htm.

Guzmán, A., y Alvarado J. (2009) Fases y Operaciones Metodológicas de Investigación Educativa. Asociación de Investigadores en Ciencias de la Educación. México.

Hernández y Pérez (2011). Evaluación de la docencia a nivel universitario. Memorias del XI Congreso Nacional de Investigación Educativa. COMIE. Colima, México.

Lule, M. (2003). Analizar la práctica docente con dimensiones constructivistas: una experiencia de observación. Universidad Veracruzana. Xalapa. México. En: www.uv.mx/facpsi/revista/documents/lulu.pdf

Martínez I. (2011). Los criterios de evaluación como detonante programación didáctica. Revista Avances en Supervisión educativa. No. 14. En www.adide.org.

Monroy, M. (1988). El pensamiento didáctico del profesor: un estudio con profesores de ciencias histórico sociales del colegio de bachilleres y del Colegio de Ciencias y Humanidades. Tesis. Facultad de Psicología. UnAm. México

Ortega, F.; Rosales, R. y Sánchez B. (2011). XI Congreso Nacional de Investigación Educativa. Sujetos de la Educación Ponencia. En www.comie.org.mx/congreso/memoriaelectronica/v11/docs/.../0532.pd

Prieto L. (2005). Las creencias de autoeficacia docente del profesorado universitario. Universidad Pontificia Comillas. Madrid.

Prieto, L. (2007). Autoeficacia del profesor universitario. Eficacia percibida y práctica docente. Narcea. España.

Programa institucional de formación docente (2012). Versión actualizada por la dirección general de desarrollo académico. 